\title{
An Improved Quantum Particle Swarm Algorithm for Routing Optimization of Wireless Sensor Networks
}

\author{
Xing Jin \\ Rizhao Polytechnic, Rizhao, China \\ Received: July 17, 2020. Revised: December 23, 2020. Accepted: January 18, 2021. Published: January 27, 2021.
}

\begin{abstract}
Because of the limited energy of wireless sensor nodes, the energy loss of communication process affects network performance. In order to prolong the network lifetime and combine the fast ergodicity of the quantum particle swarm, this paper proposes a wireless network routing optimization method to improve the quantum particle swarm, aiming at the leach problem of the wireless sensor network WSN Classic Clustering protocol. The optimal routing speed is accelerated, and the optimal routing and energy consumption of the network are balanced as far as possible, and adjust the parameters of the cluster head election threshold to obtain the most suitable clustering structure for the current environment adaptively, so as to balance the network energy consumption and improve the network life. The experimental results show that compared with the LEACH-EP protocol, GA and PSO, the proposed protocol has better performance in network lifetime and network latency.
\end{abstract}

Keywords - circuits, systems, signal processing, wireless sensor network, quantum particle swarm algorithm, path optimization

\section{INTRODUCTION}

$\mathrm{W}$ IRELESS sensor networks, which are composed of several sensor nodes with the capability of communication, perception and data processing, have been widely used in the fields of industrial production, environmental monitoring, intelligent building, medical health and military, and play an important role in the Internet of things[1-3]. Because of its multidisciplinary intersection and integration of various techniques, it has aroused great concern in the Frontier hot research field. Wireless sensor networks are mainly composed of a large number of sensor nodes to complete data collection, transmission and processing. Because the wireless sensor network node's hardware resources and the energy limited, the data processing and the transmission ability is weak, the sensor node's energy mainly relies on itself carries the battery to supply and the capacity is limited, it is difficult to replace the battery, causes the node energy to be limited and cannot supplement. For wireless sensor networks with limited energy, reducing energy consumption is an important measure to prolong the network life [4].

Sensor nodes can be used for data collection, data forwarding and information processing. Sensors, microcontrollers and transceivers are the main components of such sensor networks. The sensor is responsible for converting the physical data into electrical signals, and then it transmits the collected data to the microcontroller, which is responsible for processing the acquired sensing data and controlling the wireless communication system. Transceivers allow data communication with other nodes in the network or with base stations. Network radio transmission is realized by using media access control (MAC) protocol. What all three components have in common is the power distribution unit that determines the power distribution scheme of the three components. How to use energy effectively, prolong the life of wireless sensor network, design a special routing protocol for Wireless sensor network, and ensure that network function has become an important direction in the research of Wireless sensor Network routing protocol, has very important significance.

The structure of this paper is as follows. In Section 2 we give a review on the Wireless Sensor Network Path Optimization algorithms. Section 3 presents our system model and we discuss our proposed method in Section 4. Extensive simulation results and analysis are given in Section 5. Finally, we conclude in Section 6. 


\section{RELATED WORKS}

The structure of sensor module is as follows: Memory, Battery, Sensor Hardware, Embedded Processor and Transceiver. The size of wireless sensor platform mainly depends on their batteries, usually two AA batteries. In terms of energy efficiency, the platform based on TIMSP430 Microcontroller is at the most advanced level of technology, and the current consumption in full working mode is about $10 \%$ The current consumption in idle mode is less than $1 \mu \mathrm{A}$.

The second key factor to be considered in signal processing and data processing technology of wireless sensor networks is the communication between modules. Communication leads to two key aspects, which may damage the theoretical validity of data processing algorithms in wireless sensor networks First of all, we can not assume that the network connectivity is perfect, and link change should be a feature of the simulation framework. Secondly, the synchronization from code execution to network communication should also be considered, because they play a major role in the actual deployment of sensor networks. There are various kinds of sensor network simulation systems from sensor module microcontroller to network communication For example, from a network perspective, NS2 is a prominent simulator, which can accurately simulate wired and wireless networks with thousands of nodes. Although it is beneficial to a wide range of standard and experimental communication protocols and network architecture, it lacks the simulation of code execution on network nodes. ATEMU and AVRORA provide cycle accuracy in machine code level simulation of sensor module platform based on AVR assembly language, and provide network simulation of hundreds of nodes. However, it is limited to AVR Microcontroller, and can not simulate the code execution of other types of microcontrollers. Other projects such as EmStar or SENS, which can simulate the interaction in heterogeneous networks by focusing on higher-level interoperability issues, involving low resource sensor module and higher resource micro server platform.

At present, TOSSIM, or TinyOS simulator, is the best choice to simulate particle code execution and network communication with reasonable accuracy. TinyOS is an open source event driven system, which is used to program the current main types of sensor modules. It has the advantages of a large number of program contributions and a large number of users. The program language is a variant of $\mathrm{C}$ language $\mathrm{NesC}$, can be compiled as TI MSP430 and ATmega128L AVR microcontrollers, these microcontrollers are currently most widely used in sensor module technology. TOSSIM simulates the actual code running on the sensor module at $4 \mathrm{MHz}$ through the virtual clock. It also reproduces the complete network stack of TinyOS at bit level, so as to simulate network contention or packet damage through simple mechanism. Finally, it provides a simple generation of simplified connection network model and energy consumption model.

For the optimization of energy utilization efficiency and energy balance in wireless sensor networks, the existing literatures are studied and solved from many aspects. The typical clustering protocol for prolonging network life is: Low power adaptive layered Clustering (leach) protocol, however, the protocol has many drawbacks.

For this, the current clustering protocol is mainly aimed at the cluster scheme and cluster selection mechanism.

The clustering algorithm is improved by using swarm intelligence algorithm and fuzzy algorithm respectively, so as to optimize energy consumption and enhance network performance [5-6]. An energy-aware Leach protocol is proposed: LEACH-EP, in which nodes with more energy have more opportunities to become cluster Heads $(\mathrm{CH})$ and calculate energy thresholds based on the expected node's percentage of $\mathrm{CH}$, the current remaining energy and the average remaining energy of all CHS in the previous round. This protocol increases the network lifetime by $33 \%$ than Leach [7]. In paper [8], a genetic algorithm (GA) is proposed to optimize WSN, the initial cluster head table is constructed by Leach algorithm, and the approximate optimal solution is found by using GA for efficient searching, which can be used to improve the life of WSN, but it is easy to get into the local optimal solution. The cluster head is elected according to the residual Energy, node connectivity and the total time of the elected $\mathrm{CH}$, and the simulated annealing (SA) algorithm is used to optimize all the nodes until the energy consumption of all the clusters is nearly balanced. However, the SA has been searching globally for a long time [9]. The paper [10] adopts the scheme of Mobile base station node to optimize network energy consumption and solve the problem of energy void.

However, the scheme is only suitable for fixed network applications, and the change of Base station node location will result in the increase of routing path update cost of network nodes. Yessad presents a Multipath routing protocol, in which nodes calculate the choice probability of different routes according to the residual energy of different routing paths, the communication energy and the number of forwarding nodes in the path, in order to realize the efficiency and equalization of network energy consumption and improve the whole life of the network [11]. However, the calculation of routing probability of this method requires a large computational overhead. In the paper [12], an energy balance routing protocol based on potential field theory is proposed, and the virtual potential field is established by the potential field theory to ensure that the data packet is transmitted to the base station through high energy region to protect the nodes with low residual energy. The article [13] analyzes the network attack behavior which is related to the concept of security trust, and makes a simple classification, but does not propose the corresponding plan. In [14], a trust calculation method based on D-S evidential theory is proposed.

The method can be used to deal with the information of evidence, obtain the input of trust calculation, and guarantee the precision of trust calculation, but its computational complexity is high, and additional data storage resource cost is needed. 
Literature [15] based on game theory, this paper analyzes the relationship between network collaboration, trust and security, focuses on the modeling of trust calculation, and does not design trust data acquisition process. In the paper [16], a secure routing protocol (TSR) for on demand unicast is proposed. Based on the historical behavior record of the nodes, a simple trust Prediction model is established, and the safe route is chosen according to the result of trust evaluation. However, the protocol only evaluates the direct trust evaluation of a node as a trust value, which results in a one-sided and inaccurate trust value calculation.

In recent years, researchers have carried out a lot of research on the applications of Neural Network algorithm to wireless sensor networks. A neural network model is proposed to find the lowest weak connected dominating set in wireless sensor networks, and a new direct fusion algorithm can be chosen to select a suitable transmission radius. Stabilize the network and extend the life cycle of the network [17]. In the paper [18], a Markov model is established using a back propagation (BP) neural network to compute the life cycle of wireless sensor networks, which accurately gives the maximum life cycle value and reduces computational complexity. In the paper [19], a neural network fusion algorithm based on principal element Analysis (PCA) is proposed to process the collected data in the clustering structure of wireless sensor networks, which reduces the data transmission and realizes the classification of different parameter types. In the paper [20], a neural network is proposed to modify the topology of Wireless sensor network routing protocol using Neural Network to improve the efficiency of network energy utilization. In the paper [21], the neural network algorithm is applied to the energy management method of wireless sensor network, the sensor data is processed by neural network to reduce the dimension of transmission data, thus reducing the communication cost and protecting the energy. In the paper [22], for the nonlinear output of sensors affected by environmental parameters, in order to obtain accurate information to compensate for adverse environmental impacts, an effective Laguerre neural network is introduced into wireless sensor networks to make up the nonlinearity and environmental impact of sensors and reduce energy consumption.

The energy optimization algorithm mentioned above has different solutions to the energy problem, but it doesn't consider the network attack situation. The network attack of the routing layer can mislead the energy optimization routing algorithm, generate the network energy consumption and data delivery problems, and seriously affect the normal routing function of the network. However, the routing protocol based on security usually only considers the security of nodes and lacks the reasonable optimization of energy consumption.

For the network energy consumption and network security problems. In a word, when designing an efficient routing algorithm for wireless sensor networks, there are many factors that affect the performance of the network, such as minimizing total energy consumption, balancing network load, accelerating convergence and dynamic network. It would be best to consider all of these factors simultaneously to address the challenges of these real-time applications.

\section{SySTEM MODEL}

\section{A. Mathematical Model of WSN Routing}

The sensor node responsible for collecting the monitoring data from the WSN is defined as the source node. The data is transmitted to the sink through multi-hop communication. The sink fuses the data and sends the result to the base station (BS), i.e. the destination. Let WSN be abstracted into an undirected weighted graph, where $\mathrm{V}$ denotes the set of sensor nodes and $\mathrm{E}$ denotes the set of links.

$$
\begin{gathered}
V=\left\{V_{\text {head }}, v_{1}, v_{1}, \mathrm{~L} v_{n}\right\} \\
E=\left\{e_{1}, e_{2}, \mathrm{~L} e_{n}\right\}
\end{gathered}
$$

Where $V_{\text {head }}$ denotes the cluster head, and $v_{\mathrm{i}}$ denotes other sensor nodes in the cluster.

Let $\lambda_{0}$ denotes the effective transmission range of each node in $\mathrm{V}$. Then,

$$
E=\left\{e_{i} \mid D\left(v_{j}, v_{k}\right) \leq \lambda_{0}, v_{j}, v_{k} \in V\right\}
$$

\section{B. Constraints}

The operating range $d_{0}$ of route in the WSN is subject to the following constraint.

$$
d\left(v_{i}, v_{j}\right) \leq d_{0}
$$

The energy consumption $\hat{E}$ associated with transmission paths throughout the WSN is to be minimized.

$$
\min (\hat{E})=\sum_{i, j} \lambda d\left(v_{i}, v_{j}\right)^{2}
$$

where $\lambda$ denotes the energy consumption coefficient. Considering the need for energy consumption balance between network nodes, the node $I$ with a lot of energy left is used to forward the data. The remaining energy index $\gamma_{i}$ is defined as: 


$$
\gamma_{i}=\frac{E_{1}}{E_{2}}
$$

Where $E_{1}$ denotes the original energy of node $i, E_{2}$ denotes the energy left with node $i$.

To avoid the low-energy nodes from being selected as the relay, it is stipulated in this paper that the node whose remaining energy is $10 \%$ of the original level is regarded as the low-energy node and will not be selected.

\section{Clustering protocol}

The LEACH protocol does not incorporate the remaining energy of node and the node position into the selection of cluster head. In this paper, the protocol is improved by taking many factors into account to compute the threshold for $\mathrm{CH}$ selection. These factors include the distance from the sink, remaining energy, number of times that the node is selected as the $\mathrm{CH}$ previously, and the distance from other cluster heads, which are called sub-threshold terms and represented with T1$\mathrm{T} 4$, sequentially. The threshold $\mathrm{T}_{\mathrm{ASLPR}}(\mathrm{n})$ for $\mathrm{CH}$ selection is computed as:

$$
T_{\text {ASLPR }}(n)= \begin{cases}Z(n) & E(n) \geq t_{1} \frac{1}{N} \sum_{i=1}^{N} E(i) \\ 0 & E(n)<t_{1} \frac{1}{N} \sum_{i=1}^{N} E(i)\end{cases}
$$

where $Z(n)=\alpha_{1} T_{1}(n)+\alpha_{2} T_{2}(n)+\alpha_{3} T_{3}(n)+\alpha_{4} T_{4}(n)$ , $\alpha_{1}, \alpha_{2}, \alpha_{3}, \alpha_{4}$, are the weight of each sub-threshold term and $\sum_{i=1}^{4} \alpha_{i}=1, t_{1}$ denotes a threshold parameter.

\section{IMPROVED QUANTUM PARTICLE SWARM OPTIMIZATION} (QPSO) ALGORITHM

Particle swarm optimization (PSO) algorithm is a kind of adaptive probabilistic optimization technique for simulating swarm intelligence behavior for complex system computation. When PSO solves the optimization problem, each particle has its own position and velocity (distance and direction), the position of the particle is a solution to the optimization problem, through the particle position coordinates to calculate the fitness value to determine the position of the pros and cons of the search process in the past, the optimal position of each particle is called the individual extreme value, namely Pbest The optimal position of all particles is called global extremum, i.e. gbest.
In order to find the optimal solution of the problem, the particle keeps updating its position in the solution space by tracking the two extremum by the velocity and position update formula. The particle swarm optimization algorithm uses certain evolutionary rules to solve in the search space, with the memory, the particle information is shared in the whole population, but in the particle swarm algorithm, in addition to its own memory pbest, only the global optimal particle gbest share information, so the particle swarm optimization group particle can move to the optimal solution, so that it converges faster. At the same time, such a mechanism may lead to too much concentration of population particles in particle swarm optimization, which makes the algorithm fall into local optimum.

In this paper, particle swarm algorithm has better convergence speed and simpler algorithm, it is easy to fall into the local optimal defect for particle swarm optimization, and proposes an improved strategy of quantum particle swarm optimization. The concept of quantum computing was first proposed by R.landauer and C.bennett in the 70 's, and quantum computing has the characteristics of parallelism and digital storage, and has powerful computing power. Quantum particle swarm optimization (PSO) combines the idea of particle swarm optimization and the quantum computation method, the position of the quantum particle is encoded by the quantum bits, the particle position is updated by the Quantum revolving gate, the randomness of the particle position is greatly increased, and it is easier to appear in the whole solution space, and the whole feasible solution space can be searched. It has stronger global optimization ability and overcomes the defects of standard particle swarm optimization.

The quantum position of a quantum particle is represented by a quantum bit, and the quantum position is defined as $\left(\begin{array}{llll}\alpha_{1} & \alpha_{2} & \ldots & \alpha_{1} \\ \beta_{1} & \beta_{2} & \ldots & \beta_{1}\end{array}\right)$, In which $\left|\alpha_{j}\right|^{2}+\left|\beta_{j}\right|^{2}=1,(j=1,2, \ldots, l)$. In order to make the algorithm more concise and effective, set $0 \leq \alpha_{j} \leq 1,0 \leq \beta_{j} \leq 1$, and $\beta_{j}=\sqrt{1-\alpha_{j}^{2}}$. Thus, the quantum position of the first quantum particle can be simplified as

$$
\mathbf{x}_{\mathrm{i}}=\left(\begin{array}{llll}
\alpha_{\mathrm{i} 1} & \alpha_{\mathrm{i} 2} & \ldots & \alpha_{\mathrm{i} 1}
\end{array}\right)=\left(\begin{array}{llll}
\mathrm{x}_{\mathrm{i} 1} & \mathrm{x}_{\mathrm{i} 2} & \ldots & \mathrm{x}_{\mathrm{i} 1}
\end{array}\right)
$$

The update of quantum particle position in quantum particle swarm optimization using the quantum revolving gate, defined as $R\left(\theta_{i j}^{k}\right)=\left(\begin{array}{cc}\cos \theta_{i j}^{k} & -\sin \theta_{i j}^{k} \\ \sin \theta_{i j}^{k} & \cos \theta_{i j}^{k}\end{array}\right)$, using the quantum revolving gate $R\left(\theta_{i j}^{k}\right)$ to update the J-Qubit of the first quantum particle $x_{i j}$ can be expressed as 


$$
\left.\mathrm{x}_{i j}^{k+1}=\left|R\left(\theta_{i j}^{k}\right) \mathrm{x}_{i j}^{k}\right|=\mid \begin{array}{cc}
\cos \theta_{i j}^{k} & -\sin \theta_{i j}^{k} \\
\sin \theta_{i j}^{k} & \cos \theta_{i j}^{k}
\end{array}\right) \mathrm{x}_{i j}^{k} \mid
$$

Similarly, the formula can be simplified to

$$
\mathrm{x}_{i j}^{k+1}=\left|\mathrm{x}_{i j}^{k} \times \cos \theta_{i j}^{k+1}-\sqrt{1-\left(\mathrm{x}_{i j}^{k}\right)^{2}} \times \sin \theta_{i j}^{k+1}\right|
$$

Quantum bits $\mathrm{x}_{i j}^{k}$ are updated with quantum non gate $\mathrm{v}$ when the quantum rotation angle is $\theta_{i j}^{k}=0$ used, and the update process can be described as:

$$
\mathrm{x}_{i j}^{k+1}=\sqrt{1-\left(\mathrm{x}_{i j}^{k}\right)^{2}}
$$

In quantum particle swarm optimization, the update of the quantum rotation angle and position of the $\mathrm{D}$ dimension of the first quantum particle is corresponding to the update of particle velocity and position in the particle swarm, and the update process can be expressed as follows:

$$
\begin{gathered}
\theta_{i d}^{k+1}=c_{1} \cdot \varepsilon_{1} \cdot\left(P_{i d}^{k}-x_{i d}^{k}\right)+c_{2} \cdot \varepsilon_{2} \cdot\left(P_{g d}^{k}-x_{i d}^{k}\right) \\
x_{i d}^{k+1}=\left\{\begin{array}{cc}
\sqrt{1-\left(x_{i d}^{k}\right)^{2}}, & \theta_{i d}^{k+1}=0 \\
\left|x_{i d}^{k} \times \cos \theta_{i d}^{k+1}-\sqrt{1-\left(x_{i d}^{k}\right)^{2}} \times \sin \theta_{i d}^{k+1}\right|, & \theta_{i d}^{k+1} \neq 0
\end{array}\right.
\end{gathered}
$$

Where $1 \leq i \leq m, 1 \leq d \leq 5, \mathrm{~K}$ and $\mathrm{k}+1$ represent the number of iterations, $\theta_{i d}^{k}$ is the quantum rotation angle of the first $\mathrm{d}$ quantum particle in the $\mathrm{K}^{\text {th }}$ iteration. $\varepsilon_{1}$ and $\varepsilon_{2}$ is the 0 mean variance is 1 of the Gaussian distribution of the random number, $c_{1}$ and $c_{2}$ determines the quantum particle of the individual optimal solution and the entire quantum particle swarm of the global optimal solutions to the quantum particle position of the update process influence degree, $x_{i d}^{k}$ is the position of the first particle in the $\mathrm{D}$ dimension of the $\mathrm{K}$ iteration, $P_{i d}^{k}$ is the position of the individual extreme point in the first $\mathrm{D}$ dimension of the first $\mathrm{k}$ iteration, which is the position of the global extremum point of the whole particle swarm in the $\mathrm{K}^{\text {th }}$ iteration of the first D dimension.

In the process of optimization, the selection, crossover and mutation of genetic algorithm are used to speed up the convergence speed and improve the ability of global search, and finally get an optimal data transmission path.

\section{RESULTS AND DISCUSSIONS}

In this simulation experiment, the network coverage range is $100 \mathrm{mx} 100 \mathrm{~m}$ rectangular plane area, randomly distributes 50 sensor nodes, and the nodes are numbered uniformly. The initial energy of the node is $0.5 \mathrm{~J}$, the effective transmission distance between nodes is $10 \mathrm{~m}$, the total number of ants selected in the experiment is 80 , the crossover probability is 0.8 , the initial energy of each node is $110 \mathrm{~J}$, the energy consumption of the transmit/receive 1 bit data is $0.05 \mathrm{j} / \mathrm{bit}$, the maximum iteration number is 100 . The genetic algorithm (GA), particle swarm optimization (PSO), LEACH-EP protocol and the research algorithm presented in this paper are compared in the simulation experiment. Under the condition that the test environment parameters are the same, each algorithm runs 20 times Monte Carlo Test, taking the average value as the final result.

\section{A. Performance Evaluation Index of Algorithm}

In order to better see the optimization efficiency of each algorithm and make the result comparable, the performance evaluation index of the algorithm needs to be established. This index is completed in two aspects from path energy consumption and the success rate of finding the optimal path. In the process of finding the optimal path, it is necessary to consume energy continuously, and the total energy consumed is:

$$
\mathrm{E}=\sum_{i=1}^{\mathrm{n}} e_{i}
$$

Where $E$ represents the total energy consumed, and EI represents the energy consumed by each node. The energy consumption in the transmission process is calculated by free space model.

The path loss is calculated by the following formula

$$
\begin{gathered}
\lambda=\frac{C}{\text { Bandwi dt } h} \\
e_{i}=\frac{\lambda^{2}}{4 \pi^{2} d^{2}}
\end{gathered}
$$

Therefore, the residual energy of each node can be calculated under the precondition of the initial energy, the number of simulation times and the running time of the sensor. 


\section{B. Energy consumption Simulation Results}

The simulation results of the total energy consumption of 4 algorithms, as shown in Fig.1, show that the improved quantum particle swarm optimization algorithm in this paper consumes the least energy in the process of finding the optimal path, followed by the particle swarm algorithm, and the LEACH-EP protocol consumes the most energy. Therefore, the QPSO algorithm can reduce the energy consumption and prolong the life cycle of the whole network.

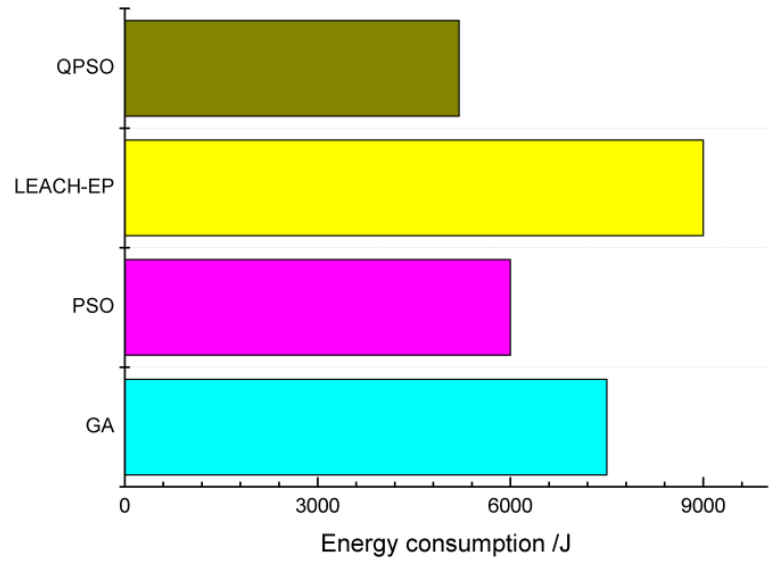

Fig. 1 Algorithm energy consumption comparison

\section{Comparison and analysis of network survival time}

The network survival Time simulation results of 4 algorithms are shown in Figure 2. According to the results of Fig. 2, the network survival time of this algorithm is much larger than that of genetic algorithm ( GA ), PSO algorithm and LEACH-EP, and the lifetime of wireless sensor network decreases gradually with the number of perceived nodes decreasing, but the algorithm is slower than other algorithms to reduce the speed. Can effectively extend the lifetime of the entire wireless sensor network.

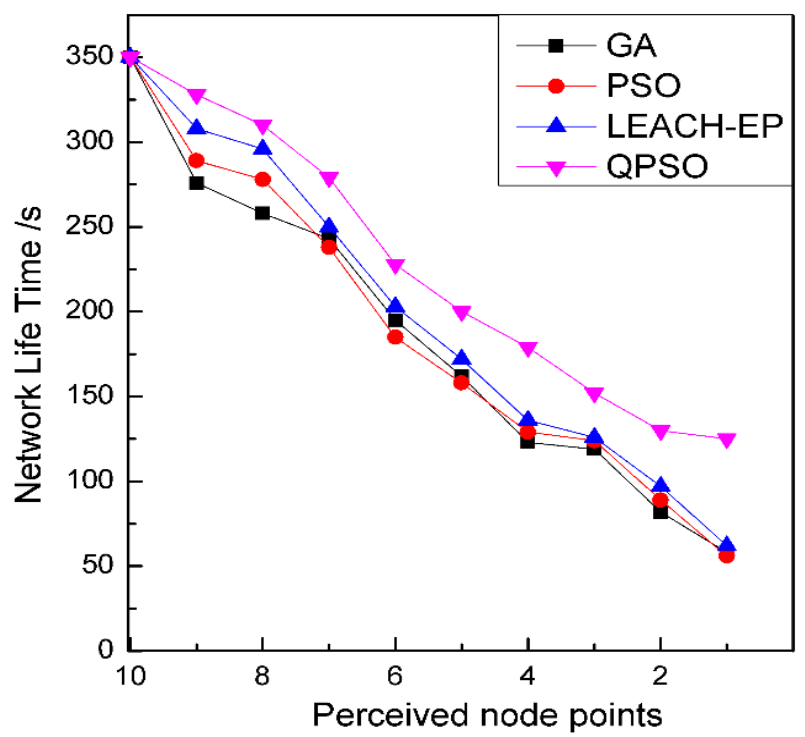

Fig.2 Comparison of perceived survival time

Table 1 shows the network lifetime data (rotations) for the 4 protocols at FND, HND, and Lnd, which is the average number of experiments running 10 times. We can see that, compared with GA, PSO and LEACH-EP, the life span of the FND of this Protocol is increased by $114.9 \%, 55.8 \%$ and $33.2 \%$, the life span of the HND of this Protocol is increased by $76.8 \%, 33.9 \%$ and $18.2 \%$, and the life span of the LND of this Protocol is increased by $34.8 \%, 19.8 \%$ and $11.6 \%$.

Table 1. The network life data of 4 protocols

\begin{tabular}{|l|l|l|l|}
\hline Protocols & FND & HND & LND \\
\hline GA & 528.4 & 725.8 & 1025.6 \\
\hline PSO & 728.8 & 958.6 & 1153.8 \\
\hline LEACH-EP & 852.3 & 1085.9 & 1238.5 \\
\hline QPSO & 1135.4 & 1283.6 & 1382.7 \\
\hline
\end{tabular}

\section{CONCLUSION}

In order to solve the problem of data transmission energy consumption in wireless sensor networks and how to recover the path quickly when a dead node appears on the transmission path, a wireless sensor routing optimization algorithm based on improved quantum particle swarm algorithm is proposed in this paper. Considering various node factors, the optimal clustering structure of the network is obtained.

Compared with the existing algorithms based on genetic optimization and PSO, the simulation shows that the algorithm makes the residual energy difference between nodes in wireless 
sensor network is the least, and the average residual energy of different node packets is more, which can prolong the lifetime of the wireless sensor network effectively. But the link quality is not considered in the algorithm, network latency and other factors related to QoS, with the increase of node radius, the number of neighbor nodes increases, and the burden of the nodes will increase, therefore, in the subsequent research work, based on this algorithm, how to optimize the experimental parameters to improve the performance of the algorithm, Still need further study.

\section{REFERENCES}

[1] AlKaraki, Jamal N, Kamal, Ahmed E. "Routing techniques in wireless sensor networks: a survey". IEEE Wireless Communications, vol.11, no.6, pp.6-28, 2009.

[2] I.F. Akyildiz, W. Su, Y. Sankarasubramaniam, et al. "Wireless sensor networks: a survey". IEEE Communications Magazine, vol.38. no.4, pp. 393-422, 2002.

[3] Q. Cao, T. He and T. F. AbdelZaher, "uCast: Unified Con- nec-tionless Multicast for Energy Efficient Content Distribution in Sensor Networks", IEEE Transactions on Parallel and Distri-buted Systems, vol. 18, no. 2,, pp.240-250, 2007.

[4] B. Aoun and R. Boutaba, "Clustering in WSN with Latency and Energy Consumption Constraints," Journal of Network and Systems Management, vol. 14, no. 3, pp. 415-439, 2006.

[5] Karaboga D, Okdem S, Ozturk C. "Cluster based wireless sensor network routing using artificial bee colony algorithm", Wireless Networks, vol.18, no.7, pp.847-860. 2012.

[6] Mao, S., Zhao, C., Zhou, Z. et al. "An Improved Fuzzy Unequal Clustering Algorithm for Wireless Sensor Network", Mobile Networks and Applications, vol.18, no.2, pp. 206-214, 2013.

[7] Fengjun Shang, Yang Lei. "An Energy-Balanced Clustering Routing Algorithm for Wireless Sensor Network", Wireless Sensor Network, vol.135, no.2, pp.777-783, 2010.

[8] R. Kumari and R. Kumar, "An energy efficient hybrid optimized routing protocol for WSN," In International Conference on Telecommunication and Networks (TEL-NET), Noida, 2017, pp. 1-6.

[9] Azami, Mostafa .R, Manij .S, et al. (2013). "Increasing the Network life Time by Simulated Annealing Algorithm in WSN with Point", International Journal of Ad hoc, Sensor \& Ubiquitous Computing, vol. 4, no.2, pp.31-45, 2013.

[10] Shuvo S , Hossain E, Khan Z R . Fixed Point Implementation of Grid Tied Inverter in Digital Signal Processing Controller. IEEE Access, 2020, 8:89215-89227.

[11] Cox M, Thijs V D L, De Vries B . A factor graph approach to automated design of Bayesian signal processing algorithms. International Journal of Approximate Reasoning, 2019, 104:185-204.

[12] F. Ren, J. Zhang, T. He, C. Lin and S. K. D. Ren, "EBRP: EnergyBalanced Routing Protocol for Data Gathering in Wireless Sensor Networks," IEEE Transactions on Parallel and Distributed Systems, vol. 22, no. 12, pp. 2108-2125, 2011.

[13] YU Y, LI K, ZHAO W, et al. "Trust mechanisms in wireless sensor networks: Attack analysis and countermeasures" Journal of Network and Computer Applications, vol. 35, no.3, pp. 867-880, 2012.

[14] Md. Arafatur Rahman, "IoT Enabled Intra-Vehicular Wireless Sensor Networks for Reliable Communications," WSEAS Transactions on Communications, pp.101-110, Volume 17, 2018.

[15] Vinayak Musale, Devendra Chaudhari, "Design Issues, Principles and Routing Challenges in Sensor Networks," WSEAS Transactions on Computers, pp.247-252, Volume 17, 2018.

[16] XIA H, JIA Z, LI X, et al. "Trust prediction and trust-based source routing in mobile ad hoc networks", Ad Hoc Networks, vol. 11, no. 7, pp. 20962114, 2013.

[17] He H, Zhu Z, Kinen E. "A neural network model to minimize the connected dominating set for self-configuration of wireless sensor networks", IEEE Transactions on Neural Networks, vol.20, no.6, pp.973999, 2009.
[18] Yang W, Wang B, Liu Z, et al. "Back Propagation Neural Network Based Lifetime Analysis of Wireless Sensor Network", Advances in Neural Networks, Springer Berlin Heidelberg, pp.956-962, 2009.

[19] Feng X, Xu Z. "A Neural Data Fusion Algorithm in Wireless Sensor Network", In Pacific-Asia Conference on Circuits, Communications and Systems. 2009, pp.54-57.

[20] Patra C, Guha A, Chattopadhyay A. "Energy-Efficient Topologies for Wireless Sensor Network: Neural Approach", International Journal of Distributed Sensor Networks, 2010, doi:10.1155/2010/216716

[21] Enami N, Moghadam R A, Haghighat A. "A Survey on Application of Neural Networks in Energy Conservation of Wireless Sensor Networks", Recent Trends in Wireless and Mobile Networks, 2010, pp.283-294.

[22] Patra J C, Meher P K, Chakraborty G. "Development of Laguerre NeuralNetwork-Based Intelligent Sensors for Wireless Sensor Networks", IEEE Transactions on Instrumentation \& Measurement, vol.60, no.3, pp.725734, 2011.

\section{Creative Commons Attribution License 4.0 (Attribution 4.0 International, CC BY 4.0)}

This article is published under the terms of the Creative Commons Attribution License 4.0 https://creativecommons.org/licenses/by/4.0/deed.en_US 